\title{
Surgical techniques and functional evaluation for vestibular lesions in the mouse: unilateral labyrinthectomy (UL) and unilateral vestibular neurectomy (UVN)
}

\author{
François Simon ${ }^{1,2} \mathbb{D} \cdot$ David Pericat $^{3} \cdot$ Cassandre Djian $^{1} \cdot$ Desdemona Fricker $^{1} \cdot$ Françoise Denoyelle $^{2}$. \\ Mathieu Beraneck ${ }^{1}$
}

Received: 3 April 2020 / Revised: 26 May 2020 / Accepted: 28 May 2020 / Published online: 17 June 2020

(c) The Author(s) 2020

\begin{abstract}
Objective Unilateral labyrinthectomy (UL) and unilateral vestibular neurectomy (UVN) are two surgical methods to produce vestibular lesions in the mouse. The objective of this study was to describe the surgical technique of both methods, and compare functional compensation using vestibulo-ocular reflex-based tests.

Methods UL and UVN were each performed on groups of seven and ten mice, respectively. Main surgical landmarks were the facial nerve, the external auditory canal and the sternomastoid and digastric muscles. For UL, the sternomastoid muscle was elevated to expose the mastoid, which was drilled to destroy the labyrinth. For UVN, the bulla was drilled opened and a transcochlear approach enabled the identification of the vestibulo-cochlear nerve exiting the brainstem, which was sectioned and the ganglion of Scarpa suctioned. Behaviour and vestibular function were analysed before surgery and at 1, 4, 7 days and at 1 month postlesion using sinusoidal rotation, off-vertical axis rotation, static head tilts and angular velocity steps. Results UL is a faster and safer procedure than UVN (operative time 16.3 vs $20.5 \mathrm{~min}, p=0.19$; survival rate $86 \%$ vs $60 \%$, $p=0.25)$. UVN was more severe with significantly worse behavioural scores at day 4 and day $7(p<0.001)$. Vestibular compensation was overall similar during the first week and at 1 month (non-statistically significant difference).

Conclusion Both UL and UVN procedures can routinely be performed in the mouse with similar post-operative recovery and behavioural compensation. The operative risk of vascular or neurological damage is smaller in UL compared to UVN. UVN may be required for specific research protocols studying central cellular process specifically related to the destruction of the ganglion of Scarpa and following vestibular nerve degeneration.
\end{abstract}

Keywords Unilateral vestibular neurectomy $\cdot$ Unilateral labyrinthectomy $\cdot$ Mouse $\cdot$ Vestibular compensation $\cdot$ Surgery

Electronic supplementary material The online version of this article (https://doi.org/10.1007/s00415-020-09960-8) contains supplementary material, which is available to authorized users.

François Simon

f.simon@aphp.fr

$\bowtie$ Mathieu Beraneck

Mathieu.beraneck@parisdescartes.fr

1 CNRS, Integrative Neuroscience and Cognition Center, Université de Paris, 75006 Paris, France

2 Department of Paediatric Otolaryngology, Hôpital Necker-Enfants Malades, AP-HP.Centre, Université de Paris, 75015 Paris, France

3 Laboratoire de Neurosciences Sensorielles et Cognitives, CNRS, UMR 7260, Aix Marseille Université,

13331 Marseille, France

\section{Introduction}

The first description by Flourens in 1824 of the behavioural symptoms that follow an inner ear lesion is a starting point in the field of vestibular research [1]. The study of vestibular lesions and compensation in different vertebrate species has helped to understand vestibular physiology and the capacity of the brain to cope with the loss of a sensory function [2-10]. Unilateral labyrinthectomy (UL) and unilateral vestibular neurectomy (UVN) are two surgical methods to produce vestibular lesions. These are more invasive than pharmacological methods but are also more radical and definite lesions which may be required in certain research protocols [11]. Surgical techniques need to be described in detail to be reproducible [12-18]. Some articles report the surgical methods for UL or UVN in other animal models (e.g. frogs 
[19], chicks [20], rats [21-23], or cats [24-26]) but not in the mouse. Yet mouse models are currently the most used worldwide, due to ease of use for genetic engineering or breeding, and ethical limitations when working with larger mammals. Surgery in the mouse is challenging due to its small size and specific anatomy. Here we provide a detailed step-bystep description of both UL and UVN in adult mice. Videooculography was used to assess vestibular function following successful surgery, and to monitor vestibular compensation by quantitative measurements of the vestibulo-ocular reflex $[11,14,27]$. The objective of this study was an update of existing protocols for vestibular lesions, allowing the investigator to improve their effectiveness and safety, and reduce the duration of the surgery, critical for survival. We discuss differences in the two approaches to help choose between UL and UVN as adequate methods for a vestibular lesion.

\section{Materials and methods}

\section{Ethics statement}

A total of 17 male C57/BL6J mice, aged 10-30 weeks, was operated on, seven mice for UL and ten for UVN. Animals were used in accordance with the European Communities Council Directive 2010/63/EU. All efforts were made to minimize suffering and reduce the number of animals included in the study. All procedures were approved by the ethical committee for animal research of the University of Paris (CEEA.34).

\section{Anaesthesia and peri-operative care}

Anaesthesia and peri-operative care were identical for either UL or UVN procedure. A stock solution of anaesthesia was prepared including $1 \mathrm{ml}$ Ketamine $100 \mathrm{mg} / \mathrm{ml}$ (Virbac, Carros, France), $500 \mu 1$ Rompun ${ }^{\circledR} 2 \%$ (Bayer Vital GmbH, Leverkusen, Germany), and $8.5 \mathrm{ml}$ physiological saline solution. A dosage of $100 \mathrm{mg} / \mathrm{kg}$ ketamine and $10 \mathrm{mg} / \mathrm{kg}$ xylazine, warmed to body temperature, was administered intraperitoneally at a volume of $10 \mu \mathrm{l} / \mathrm{g}$ of body weight.

After anaesthesia, sub-cutaneous injection of buprenorphine $0.3 \mathrm{mg} / \mathrm{ml}$ (Buprecare, Axience, Patin, France) was administered at a dose of $0.08 \mathrm{mg} / \mathrm{kg}$ of body weight. Local anaesthesia using Laocaïne ${ }^{\circledR} 2 \%$ (MSD Santé Animale, Beaucouzé, France) was administered sub-cutaneously at the surgical site at a dose of $2 \mathrm{mg} / \mathrm{kg}$ of body weight.

Artificial tears (Ocry-gel, TVM lab, Lempdes, France) were administered to both eyes. Loss of pedal withdrawal reflex of both hind paws was verified before incision, and was monitored during surgery. The cervical skin immediately below the ear was shaved and cleaned (Vétédine Solution, Vetoquinol, Magny-Vernois, France). Subcutanenous hydration was performed after the surgery and twice daily for 2 days.

\section{Behaviour evaluation}

Mouse behaviour was assessed before and after surgery on day 1 , day 4 , day 7 and day 28 . Normal locomotor mouse behaviour was assessed: ability to swim (over a 30-s-long period), to groom, to move in the cage and to reach for food or water. Vestibular postural and locomotor impaired behaviour was assessed: head tilt (inclination of the head towards the lesioned side), tumbling (mouse rolling around its longitudinal axis towards the lesioned side), twirl (while the mouse is being held by the tail) and circling (stereotyped movement in circles around the mouse's hip). All eight items were quantified with a scale from 0 (normal behaviour, no deficit) to 3 (highest degree of abnormal behaviour), with a maximum deficit score of 24 .

\section{Vestibular function exploration using video-oculography}

To perform head restrained vestibular exploration, a head post was surgically implanted 2 weeks before the vestibular lesion. Head implant surgery and peri-operative care have been described previously [28, 29]. Briefly, under gas anaesthesia (isoflurane), a small custom-built head holder was cemented (C\&B Metabond) to the skull just anterior to the lambda landmark (see França de Barros et al. 2019 for a video tutorial [30]).

Vestibular function was explored before vestibular surgery and after surgery at day 1, day 4, day 7 and long-term day 28. As reported previously, [9, 28, 29, 31] all eye movements recordings were made in the dark using an infrared video system (ETL-200, ISCAN, Burlington, MA, USA), recording pupil and corneal reflection (CR) position.

Eye movements were recorded using non-invasive video-oculography [32]. The experimental set-up, apparatus and methods of data acquisition were similar to those described previously [29, 33, 34]. Briefly, mice were headfixed at a $\sim 30^{\circ}$ nose-down position to align the horizontal canals with the yaw plane [35]. Animals were placed in a custom-built Plexiglas tube secured on the superstructure of a vestibular stimulator. The VOR tests were performed in a temperature-controlled room $\left(21{ }^{\circ} \mathrm{C}\right)$ with all sources of light turned off except for computer screens. The turntable was further enclosed in a box to isolate the animal from any remaining light, with a final luminance inside the box $<0.021 x$. Myosis was induced with topical $2 \%$ pilocarpine applied $10 \mathrm{~min}$ before experimentation. Recorded eye and head position signals were sampled at $1 \mathrm{kHz}$, digitally recorded (CED power1401 MkII) using Spike 2 software 
and later exported into the Matlab programming environment for off-line analysis (Matlab, The MathWorks).

i. Videonystagmography first recorded spontaneous eye movements without any vestibular stimulation, and number and direction of the nystagmus rate per minute were reported.

ii. Then, the angular horizontal vestibulo-ocular reflex (aVOR) was tested during horizontal sinusoidal rotation of the turntable (at $0.2 ; 0.5 ; 1$ and $1.5 \mathrm{~Hz}$; peak velocity $30 \%$ s). Analysis was made on at least 10 cycles. Two parameters were extracted from the recordings: the gain and the phase. The gain was the ratio between the amplitude of the eye (response) and head (stimulus) rotations. Since the animal was headfixed to the rotating table, head movements and table movements were identical. The phase was the temporal shift between the eye and table rotations, expressed in degrees as the ratio of the sinusoidal cycle (2 pi). Details for gain and phase calculation were reported in Carcaud et al. [33]. Values with VAF (varianceaccounted-for) under 0.5 were discarded [28].

iii. The eye movements evoked by a specific stimulation of the otolith organs (maculo-ocular reflexes, MOR) were tested [36] using an off-vertical axis rotation (OVAR) as previously described [29]. Briefly, the axis of rotation was tilted from the vertical by $17^{\circ}$. Rotations were performed at a constant speed $(50 \%)$ for at least 10 rotations both in clockwise $(\mathrm{CW})$ and counterclockwise (CCW) directions. Due to the inertial nature of the angular movement detection, a rotation at constant speed elicits a combined canalar and otolithic response at the onset of movement, however, after a few seconds only the otolithic component remains $[29,37]$. Since gravitational acceleration acts vertically, this stimulation is equivalent to a continuous rotation (at $0.14 \mathrm{~Hz}$ ) around the mouse's head of a $17^{\circ}$ tilted constant linear acceleration stimulus (see Fig. 2b in Beraneck et al. [29]). For horizontal OVAR responses, quick-phases of reflexive eye movements were identified and not considered for analysis. During rotations, the velocity of horizontal slow phases is modulated (modulation, $\mu$ ) around a constant bias $(\beta)$. Both parameters $(\mu$ and $\beta)$ were calculated from the sinusoidal fit of eye horizontal slow-phase velocity using the least-squares optimization of the equation:

$S P(t)=\beta+\mu \bullet \sin \left[2 \pi \bullet f_{0} \bullet\left(t+t_{d}\right)\right]$

where $\mathrm{SP}(t)$ is the slow-phase velocity, $\beta$ is the steadystate bias slow phase velocity, $\mu$ is the modulation of eye velocity, $f_{0}$ is the frequency of table rotation, $t_{d}$ is the dynamic lag time (in msec) of the eye movement with respect to the head movement. The bias (Maculoocular reflex Bias; $\mathrm{MOR}_{B}$ ) is reported here as the main index of the otolithic response [29, 36]. Notably, MOR requires normal otolith function but also an efficient central velocity storage network.

iv. Static ocular counterroll (OCR) was studied: vertical pupil position according to the head tilt angle was measured first with the mouse maintained at a $0^{\circ}$ horizontal position. The platform was then tilted into different roll positions, at $10^{\circ}, 20^{\circ}, 30^{\circ}, 40^{\circ}$ and $50^{\circ}$ alternatively to the right and to the left. The platform was rotated manually and slowly to limit semi-circular canal stimulation. Measurements were made in a static position during at least $15 \mathrm{~s}$ to identify the stable pupil position. The vertical eye angle was then calculated from the raw vertical CR and pupil position [38]. The slope of a linear regression of both variables (vertical eye angle and head tilt degree) was calculated, for ispilesional $\left(-50^{\circ}-0^{\circ}\right)$ and contralateral $\left(0^{\circ}-50^{\circ}\right)$ sides.

v. Finally, angular velocity steps in the horizontal plane (hsteps) were performed at a speed of $50 \%$. The horizontal slow phase velocity decay was fitted to an exponential curve $\left(f(x)=a^{*} \exp \left(b^{*} x\right)\right)$ and the time constant $\tau$ was then calculated as $\tau=-1 / b$. The time constant of the slow phase exponential velocity decay was calculated at the start and stop of CW and $\mathrm{CCW}$ rotations. CCW-start and CW-stop, CCW-stop and $\mathrm{CW}$-start, were combined to assess left and right vestibular functions, respectively.

\section{Statistical analysis}

Statistical analysis was made using XLstats (Addinsoft, New York, NY, USA). All data are reported as mean and standard deviation. Non-parametric means were compared with the Mann-Whitney test and proportions with the Fisher test. Repeated measures ANOVA was used, three-way to compare aVOR (lesion, time and frequency), two-way for ipsilateral and contralateral OVAR, angular velocity and static headtilt stimulations and one-way for the behaviour score and nystagmus frequency count. Post-hoc comparisons were performed where appropriate using the Tukey HSD test. Values of $p<0.05$ were considered significant.

\section{Surgical techniques}

\section{Unilateral labyrinthectomy (UL) step-by-step surgical technique}

The anaesthetised mouse was put in a side-lying position. A posterior incision of the skin following the external auditory 
canal anteriorly was performed (Fig. 1). The sub-cutaneous fat was incised immediately to find the cartilaginous external

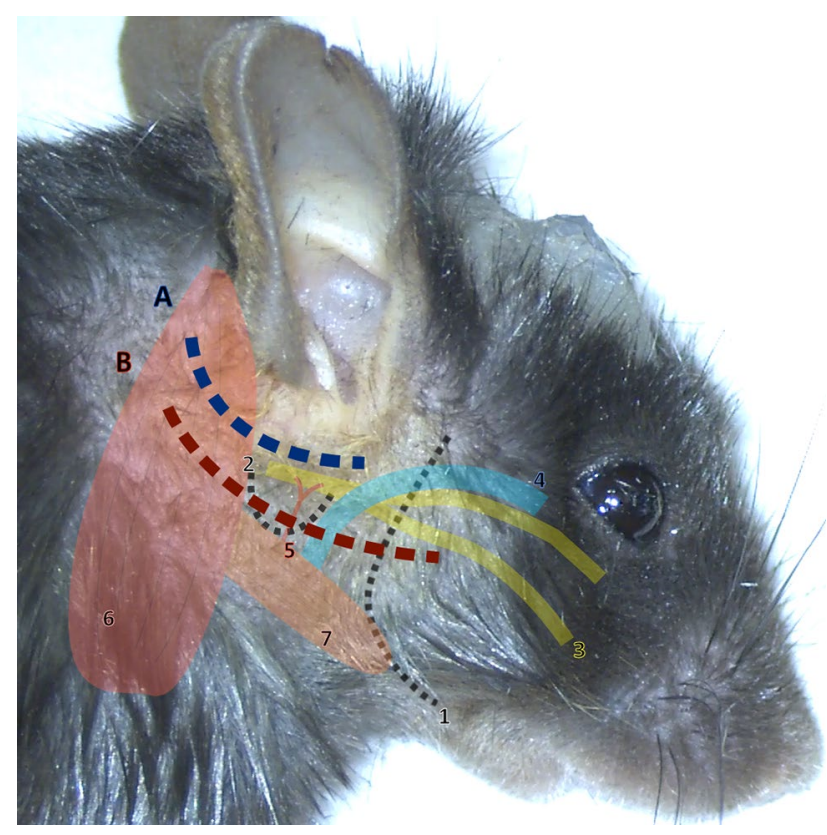

Fig. 1 Incisions and main anatomical landmarks. a (In blue): posterior incision for labyrinthectomy, $\mathbf{b}$ (in red): inferior incision for neurectomy. 1 : mandible; 2 : bulla; 3 : facial nerve; 4 : superficial temporal vein; 5: temporal artery; 6: sternomastoid muscle; 7: digastric muscle. The superficial temporal vein is one of the two main bleeding risks with the stapedial artery (not shown on this image). The mandible, facial nerve, sternomastoid and digastric muscles are key landmarks to find the bulla. The head post is visible on the mouse's skull, to be able to maintain the head in a fixed position during vestibular tests auditory canal, to dissect along with it in an avascular plane. Four retractors were positioned to open the cavity.

The first visible anatomical landmarks were the facial nerve, external auditory canal and sternomastoid muscle. The facial nerve pointed towards the mastoid which was at this stage hidden beneath the sternomastoid muscle. Thus, the sternomastoid muscle was elevated from the bone, using a cautery to prevent bleeding. The muscle was then retracted posteriorly to expose the mastoid bone (Fig. 2a).

A $0.5 \mathrm{~mm}$ cutting burr was used to drill the mastoid, immediately posteriorly to the facial nerve foramen. The bone was not be drilled too posteriorly or superiorly as the cranial cavity would otherwise have been opened.

As the posterior semi-circular canal was opened (Fig. 2b), perilymphatic liquid oozed out and was suctioned as it can sometimes impair vision. The cavity was further opened with pointed instruments such as a hook or forceps. The drill would have been dangerous at this stage as it could have damaged the stapedial artery anteriorly or breached the cranial cavity posteriorly.

The contents (utricule, saccule and cupula) were suctioned until a clear and empty vestibular cavity was visible (Fig. 2c). The ganglion of Scarpa was left intact.

An absorbable gelatin compressed sponge was packed in the cavity and the skin was closed using simple interrupted absorbable 4-0 Vicryl (Ethicon, Somerville, NJ, USA).

\section{Unilateral vestibular neurectomy (UVN) step-by-step surgical technique}

The technique was adapted from the previously published neurectomy procedure in the rat [21]. The anaesthetised
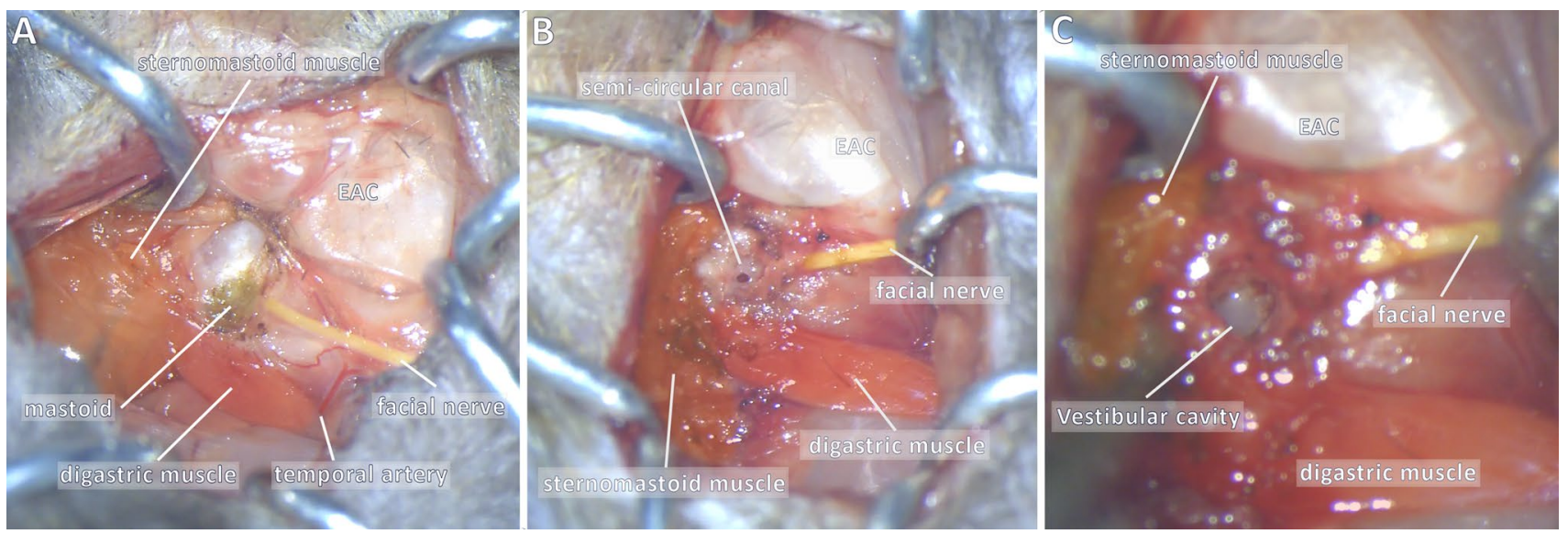

Fig. 2 Unilateral labyrinthectomy: key steps. Key steps are shown: a exposure of the mastoid bone, $\mathbf{b}$ drilling of the mastoid, $\mathbf{c}$ opening of the vestibular cavity. The mastoid bone (shown in green) can be exposed after elevation of the sternomastoid muscle posteriorly. The following anatomical landmarks stand out: sternomastoid muscle posteriorly (orange), digastric muscle inferiorly (red) and external audi- tory canal (EAC) superiorly (white). The facial nerve is of particular interest as the mastoid is immediately posterior to its foramen, and may be used as a guide to find the mastoid in an anterio-posterior direction. Great care must be taken not do damage the stapedial artery which is immediately anterior to the cavity. Full size figures are available online (Supplementary Figs. 1, 2, 3) 
mouse was put in a side-lying position. A ventral incision (Fig. 1) was performed, from the posterior limit of the external auditory canal to the mandible. After initial dissection of the subcutaneous fat, the first anatomical landmarks encountered were the jaw and masseter muscle, upon which lay the two branches of the facial nerve. Four retractors were put in place to open the surgical field. The superior branch of the nerve was parallel and close to the superficial temporal vein, which was retracted anteriorly to be protected. The superior retractor retracted the skin above the masseter, and the posterior retractor retracted the sternomastoid muscle (Fig. 3a).

The bulla was found by following the facial nerve posteriorly: the facial nerve passed horizontally between the insertion of the cartilaginous external auditory canal and the bulla.

The two major anatomical landmarks to locate the bulla were the digastric muscle inferiorly and the external auditory canal (and facial nerve) superiorly. The digastric muscle masked the posterior half of the bulla and was retracted. The bulla was also immediately beneath and slightly posterior to the Y-shaped temporal artery (Fig. 3a), which was cauterised to prevent bleeding. The bony surface of the bulla was exposed using a pointed instrument such as a hook or forceps.

The bulla was drilled using a $0.5 \mathrm{~mm}$ cutting burr, the two main vascular risks being the superficial temporal vein anteriorly and the stapedial artery in the middle ear cavity posteriorly. The bulla was cautiously opened until the stapedial artery was visualised (Fig. 3b).

Next, the cochlea was drilled with the same burr, carefully so as not to damage the stapedial artery. Perilymphatic fluid was suctioned to find the cochlear nerve. Residual bone was cleared using a hook and the cochlear nerve was then followed to reach the vestibulo-cochlear nerve and the brainstem. The VIIIth nerve was sectioned using a hook and the ganglion of Scarpa suctioned (Fig. 3c), great care was taken not to damage the brainstem underneath. The ganglion of Scarpa was visible as a bulge of the nerve, and the procedure was thus a neurectomy of the vestibular ganglion neurons (and not a neurotomy).

Gelfoam was packed in the cavity and the skin was closed using simple interrupted absorbable 4-0 Vicryl (Ethicon, Somerville, NJ, USA).

\section{Results}

A total of seven mice underwent UL and ten underwent UVN. The survival rate was higher for UL (6/7 mice, 86\%) than for UVN procedure $(6 / 10$ mice, $60 \% ; p=0.252)$. Concerning UL, one mouse did not wake up after anaesthesia, probably due to cardiogenic shock (no major bleeding during surgery). Concerning UVN, two mice died per-operatively, one immediately due to vascular damage and intense bleeding, and the other never woke up, with suspected continuous internal bleeding. The two other mice died post-operatively on day 3 and day 4, possibly due to neurological damage or cardiogenic shock. In none of the cases was there evidence for an infectious cause.

The mean age at surgery was 3.8 (range 2.5-6.3) and 4.5 (range 1.7-7.6) months, for UL and UVN, respectively. Mean operative time (from incision to closure) was $16.3 \mathrm{~min}$ (range 11-27) for UL and 20.5 min (range 18-25) for UVN, $p=0.199 .50 \%$ of UL and $100 \%$ of UVN had post-operative total right facial paralysis, although none of the nerves
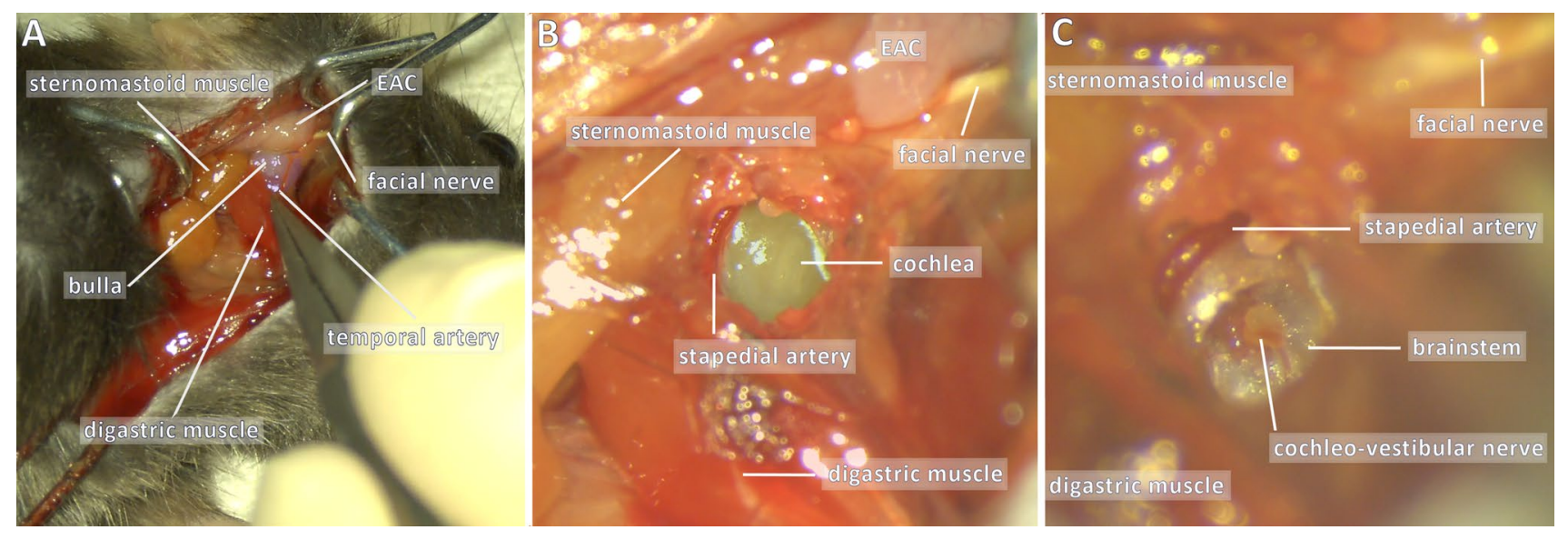

Fig. 3 Unilateral vestibular neurectomy: key steps. Key steps are shown: a exposing the bulla, b opening the bulla and $\mathbf{c}$ exposition the vestibulo-cochlear nerve. An important anatomical landmark is the facial nerve, which can be followed backwards and which runs superiorly to the bulla (purple). Other anatomical landmarks include infe- riorly the digastric muscle (red), posteriorly the sternomastoid muscle (orange) and the external auditory canal (EAC). The temporal artery also marks the bulla as it runs immediately superficially to the bulla, and must be cauterised to correctly drill the bulla. Full size figures are available online (Supplementary Figs. 4, 5, 6) 
were deliberately cut. Partial recuperation at 1 month was observed.

\section{UL and UVN clinical follow-up}

Initial clinical follow-up was identical in both UL and UVN mice. Before the animal regained full consciousness after anaesthesia, the following signs demonstrated a successful procedure: head tilted $>45^{\circ}$ and body leaning towards the operated side, also the tail $90^{\circ}$ bent towards the lesioned side. In the hours after surgery, the main behaviour indicating vestibular impairment was intense and frequent spontaneous tumbling (towards the operated side). The behavioural score was not statistically different at day 1 or after 1 month (Table 1), even though UL mice tended to recover more quickly than UVN mice (Fig. 4a). At day 4, all mice from both groups were able to reach for food and drink autonomously. The first vestibular symptom to disappear was tumbling (0/6 UL mice and $1 / 6 \mathrm{UVN}$ mice at day 4$)$. At 1 month, circling behaviour had completely disappeared in both groups, while all mice still had a noticeable head tilt $\left(<45^{\circ}\right)$ and twirled when held by the tail towards the side of the lesion. None were able to swim or float (all presented with underwater tumbling).

\section{UL and UVN functional follow-up}

Recordings of spontaneous eye movements always showed a nystagmus with the rapid beat towards the opposite side of the lesion (no nystagmus reported before surgery).

Table 1 Behavioural score and nystagmus count

\begin{tabular}{llll}
\hline Lesion & $\mathrm{UL}$ & $\mathrm{UVN}$ & $p$ \\
\hline Behavioural score & & & \\
Before & 0 & 0 & - \\
Day 1 & $21.7 \pm 1.2$ & $21.3 \pm 1.4$ & 0.522 \\
Day 4 & $\mathbf{1 1 . 5} \pm \mathbf{1 . 4}$ & $\mathbf{1 4 . 8} \pm \mathbf{0 . 8}$ & $\mathbf{< 0 . 0 0 1}$ \\
Day 7 & $\mathbf{7 . 2} \pm \mathbf{0 . 4}$ & $\mathbf{1 1 . 8} \pm \mathbf{1 . 2}$ & $\mathbf{< 0 . 0 0 1}$ \\
Day 28 & $5.0 \pm 0.6$ & $5.5 \pm 0.5$ & 0.339 \\
Nystagmus count & (beats per minute) & & \\
Before & 0 & 0 & - \\
Day 1 & $\mathbf{1 9} \pm \mathbf{1 1}$ & $\mathbf{3 1} \pm \mathbf{1 6}$ & $\mathbf{0 . 0 0 5}$ \\
Day 4 & $12 \pm 9$ & $18 \pm 12$ & 0.161 \\
Day 7 & $7 \pm 2$ & $9 \pm 5$ & 0.544 \\
Day 28 & $5 \pm 1$ & $8 \pm 3$ & 0.746 \\
\hline
\end{tabular}

All values are represented as mean $\pm \mathrm{SD}$ (standard deviation). Statistically significant values are in bold. Nystagmus count was calculated during spontaneous eye movement in the dark. Behavioural score and nystagmus count values were each compared between UL and UVN mice populations, using a two-way repeated ANOVA and post-hoc Tukey test, both model characteristics were $(40, p<0.0001)$

$U L$ unilateral labyrinthectomy, $U V N$ unilateral vestibular neurectomy
Beats/min were maximal at day 1 (statistically higher after UVN lesions) and progressively decreased (Fig. 4b) for both UL and UVN lesions (Table 1). Sinusoidal rotations at $30 \%$ s were overall not statistically different between UL and UVN lesions concerning gain or phase (Table 2). The gain values were at their lowest at day 1 with an improvement during the first week followed by a stabilisation (Fig. 4c). The gain was lower at day 28 compared to prelesion, for UL $(p=0.095, p<0.001, p=0.001)$ and for UVN $(p=0.011, p<0.001, p<0.001)$, at $0.2 \mathrm{~Hz}, 0.5 \mathrm{~Hz}$ and $1 \mathrm{~Hz}$, respectively. Phase values were not interpretable at day 1 and day 4 with $\mathrm{VAF}<0.5$, and thus were excluded from the analysis (Table 2).

Static ocular counterroll as well as responses to off-vertical axis rotation (OVAR) and angular velocity steps were analysed during rotation towards the ipsilesional and contralesional sides (Table 3). Briefly, static ocular counterroll was reduced at day 28 compared to prelesion (Fig. 4d), for tilts towards the ipsi- and contralesional side in UL mice $(p<0.001, p=0.027)$ and UVN mice $(p=0.003$, $p=0.159)$. At day 28, concerning UL, the ocular counterroll measured during inclination towards the contralesional side was statistically greater than when measured during inclination towards the ipsilesional side $(0.42 \mathrm{vs}$ $0.19, p=0.033)$. This difference in the ocular counterroll observed during rotation toward the ipsilateral or contralateral side did not reach significance for UVN ( 0.47 vs $0.34, p=0.220)$. We noted an important skew deviation (with the contralesional eye moving upwards) at day 1 and day 4 in both UVN and UL.

Concerning OVAR, MOR gain was lowest at day 1, and remained statistically lower at day 28 compared to prelesion (Fig. 4e), for rotation towards the ipsilateral side but not towards the contralesional side for UL $(p<0.001$, $p=0.746)$ and UVN $(p<0.001, p=0.729)$. At day 28 , gain for rotation towards the contralesional side was statistically higher than during rotations towards the ipsilesional side for UL (0.06 vs $0.00, p=0.011)$ and for UVN $(0.09$ vs $0.01, p<0.001)$. There was no statistically significant difference between UVN and UL mice (except at day 4 concerning rotation towards the contralesional side).

Lastly, angular velocity steps showed time constants of the velocity decay lowest at day 1 , which remained significantly lower at day 28 compared to prelesion (Fig. 4e), for both rotations towards ipsi- and contralesional sides in UL $(p<0.001, p=0.013)$ and in UVN mice $(p<0.001$, $p<0.001)$. At day 28, in UL mice, the contralesional time constant was significantly higher than the ipsilesional time constant (2.51 vs $1.21, p=0.028)$ but not for UVN (1.71 vs $1.07, p=0.277)$. There was no statistically significant difference between UVN and UL mice. 

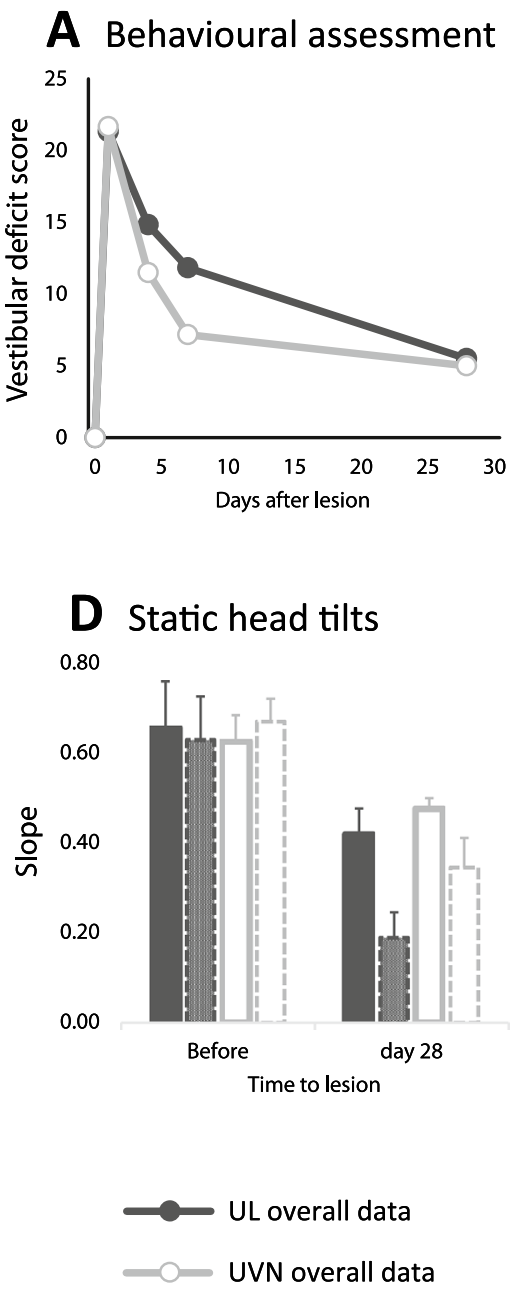

B Spont. eye movements

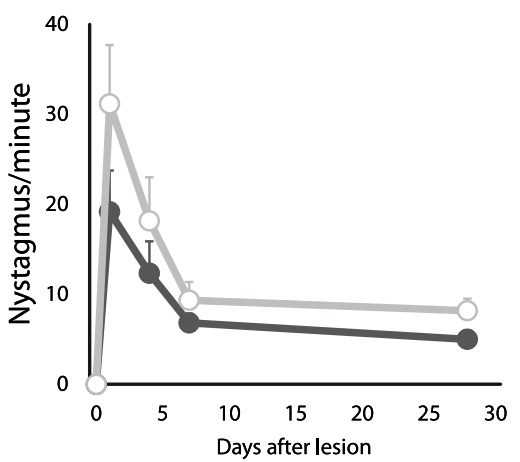

E Off Vertical Axis Rotation
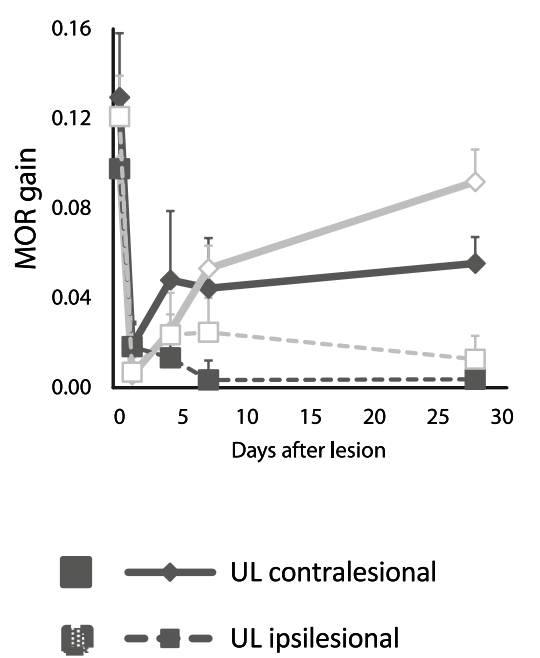

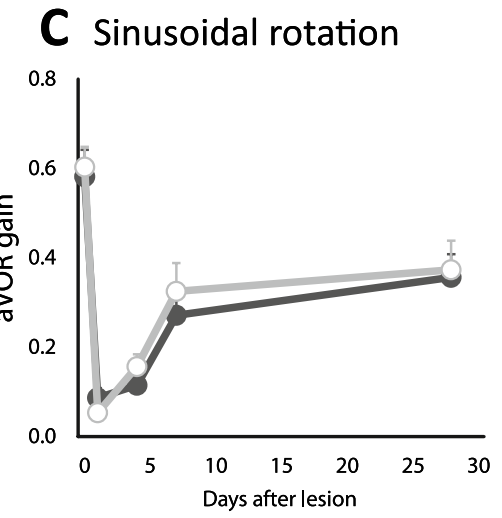

F Angular velocity steps
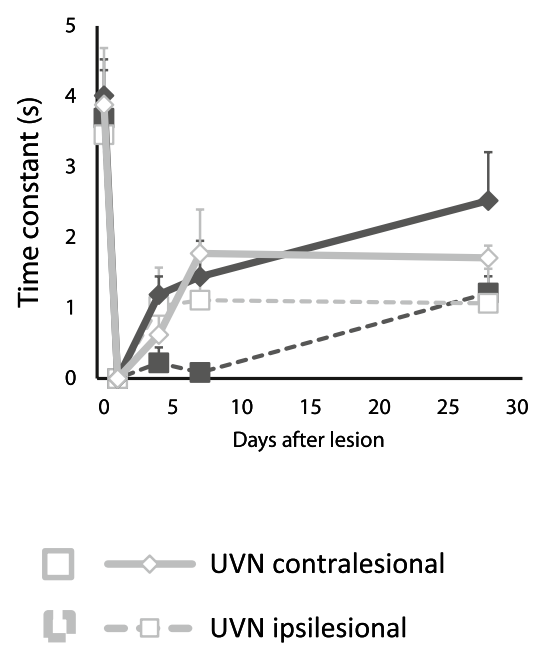

Fig. 4 Vestibular function and behaviour of unilateral vestibular neurectomy vs labyrinthectomy. All graphs show the five time points, before surgical lesion, and during follow-up at day 1, day 4, day 7 and day 28 after lesion. Points represented are mean value with SEM (standard error of the mean). Statistical comparisons are reported in Tables 1, 2 and 3. a Behavioural assessment: vestibular deficit score is represented (maximum score of 24). b Spontaneous eye movements: spontaneous recording of the pupil in the dark, reporting the number of nystagmus in $1 \mathrm{~min}$. Fast-beating component was always directed towards the contralateral side of the lesion (left side). c Sinusoidal rotation: results showing aVOR gain for a $0.5 \mathrm{~Hz}$ and $30 \%$ stimulation, demonstrating horizontal semi-circular canal function. d Static ocular counterroll: the slope of a linear equation is reported,

\section{Discussion}

Two different surgical strategies to achieve complete vestibular lesions may be used in the mouse, UL (preganglionic lesion) which destroys the peripheral labyrinthine organs and UVN (postganglionic lesion) which destroys the first order vestibular neurons in the ganglion of Scarpa (and is thus a neurectomy of the VIIIth nerve). A summary of the differences is presented in Table 4. of static lateral tilt degree and vertical position of the pupil, corresponding to utricular function. Only the reliable post-lesion results are shown at day 28. e Off Vertical Axis Rotation: clockwise and counter-clockwise rotations in yaw plane of a $17^{\circ}$ tilted platform at a rotation of speed of $50 \%$. MOR gain is reported, which corresponds to a complex integration of otolithic function and velocity storage. $\mathbf{f}$ Angular velocity steps: horizontal steps including three full rotations in yaw plane at $50 \%$ were analysed for exponential velocity decay time constant (at start and stop of clockwise and counter-clockwise rotations). Ipsilesional stimulation (right side) corresponds to $\mathrm{CW}$ start and CCW-stop, contralesional stimulation corresponds to $\mathrm{CCW}$ start and CW-stop stimulations

In both cases, surgical landmarks are key, mainly the facial nerve, the external auditory canal and sternomastoid and digastric muscles, to swiftly find the mastoid (UL) or bulla (UVN). It is also important to operate with a good quality microscope (especially for the neurectomy step) and with an electric cautery. Ketamine-xylazine general anaesthesia was preferred as isoflurane is known to induce vasodilation [39]. In addition, in the mouse, manipulation of the head during surgery may be difficult with a facial mask or intubation necessary for isoflurane. Post-operative care is 
Table 2 Canalar function (sinusoidal rotation at $30 \%$ ): aVOR gain and phase

\begin{tabular}{|c|c|c|c|c|c|c|c|c|c|}
\hline \multirow{2}{*}{$\begin{array}{l}\text { Frequency } \\
\text { Lesion }\end{array}$} & \multicolumn{3}{|l|}{$0.2 \mathrm{~Hz}$} & \multicolumn{3}{|l|}{$0.5 \mathrm{~Hz}$} & \multicolumn{3}{|l|}{$1 \mathrm{~Hz}$} \\
\hline & UL & UVN & $p$ & UL & UVN & $p$ & UL & UVN & $p$ \\
\hline \multicolumn{10}{|l|}{ aVOR gain } \\
\hline Before & $0.25 \pm 0.09$ & $0.35 \pm 0.12$ & 0.107 & $0.58 \pm 0.15$ & $0.60 \pm 0.11$ & 0.712 & $0.66 \pm 0.17$ & $0.74 \pm 0.20$ & 0.192 \\
\hline Day 1 & $0.04 \pm 0.04$ & $0.03 \pm 0.02$ & 0.865 & $0.09 \pm 0.05$ & $0.05 \pm 0.03$ & 0.570 & $0.17 \pm 0.11$ & $0.10 \pm 0.07$ & 0.294 \\
\hline Day 4 & $0.05 \pm 0.05$ & $0.07 \pm 0.06$ & 0.887 & $0.12 \pm 0.07$ & $0.16 \pm 0.07$ & 0.478 & $0.16 \pm 0.07$ & $0.29 \pm 0.09$ & 0.030 \\
\hline Day 7 & $0.12 \pm 0.08$ & $0.16 \pm 0.12$ & 0.514 & $0.27 \pm 0.12$ & $0.33 \pm 0.16$ & 0.364 & $0.42 \pm 0.21$ & $0.48 \pm 0.18$ & 0.335 \\
\hline Day 28 & $0.16 \pm 0.06$ & $0.20 \pm 0.07$ & 0.478 & $0.36 \pm 0.13$ & $0.37 \pm 0.16$ & 0.776 & $0.47 \pm 0.16$ & $0.49 \pm 0.13$ & 0.820 \\
\hline \multicolumn{10}{|l|}{ aVOR phase } \\
\hline Before & $29.3 \pm 8.3$ & $24.5 \pm 10.6$ & 0.649 & $9.9 \pm 8.7$ & $10.2 \pm 3.9$ & 0.982 & $-2.2 \pm 4.3$ & $-0.2 \pm 3.6$ & 0.855 \\
\hline Day $1^{*}$ & - & - & - & - & - & - & - & - & - \\
\hline Day 4* & - & - & - & - & - & - & - & - & - \\
\hline Day 7 & $39.6 \pm 9.4$ & $31.8 \pm 22.0$ & 0.457 & $39.2 \pm 25.6$ & $23.1 \pm 13.2$ & 0.126 & $23.2 \pm 14.6$ & $16.0 \pm 25.0$ & 0.492 \\
\hline Day 28 & $43.6 \pm 6.3$ & $26.3 \pm 41.9$ & 0.102 & $27.9 \pm 6.1$ & $25.0 \pm 11.4$ & 0.393 & $5.8 \pm 7.5$ & $6.8 \pm 4.4$ & 0.925 \\
\hline
\end{tabular}

All values are represented as mean \pm SD (standard deviation). Statistically significant values are in bold. Gain and phase values were each compared between UL and UVN mice populations, using a three-way repeated ANOVA and post-hoc Tukey test, gain model $(150, p<0.0001)$ and Phase model $(90, p<0.0001)$

aVOR angular vestibular-ocular reflex, $U L$ unilateral labyrinthectomy, $U V N$ unilateral vestibular neurectomy

*Phase was not measurable at day 1 or day 4

Table 3 Velocity storage and otolithic function

\begin{tabular}{|c|c|c|c|c|c|c|}
\hline \multirow{2}{*}{$\begin{array}{l}\text { Stimulation } \\
\text { Lesion }\end{array}$} & \multicolumn{3}{|l|}{ Ipsilesional } & \multicolumn{3}{|c|}{ Contralesional } \\
\hline & UL & UVN & $p$ & UL & UVN & $p$ \\
\hline \multicolumn{7}{|c|}{ Static head tilt, slope* } \\
\hline Before & $0.63 \pm 0.24$ & $0.67 \pm 0.12$ & 0.701 & $0.65 \pm 0.25$ & $0.62 \pm 0.14$ & 0.781 \\
\hline Day 1 & $0.16 \pm 0.26$ & $0.37 \pm 0.27$ & 0.053 & $0.57 \pm 0.20$ & $0.42 \pm 0.28$ & 0.169 \\
\hline Day 4 & $0.15 \pm 0.11$ & $0.28 \pm 0.14$ & 0.221 & $0.31 \pm 0.28$ & $0.34 \pm 0.15$ & 0.814 \\
\hline Day 7 & $0.19 \pm 0.07$ & $0.22 \pm 0.12$ & 0.811 & $0.41 \pm 0.23$ & $0.25 \pm 0.18$ & 0.144 \\
\hline Day 28 & $0.19 \pm 0.14$ & $0.34 \pm 0.16$ & 0.493 & $0.42 \pm 0.14$ & $0.47 \pm 0.06$ & 0.143 \\
\hline \multicolumn{7}{|c|}{ Off-vertical-axis rotation, MOR gain } \\
\hline Before & $0.10 \pm 0.07$ & $0.12 \pm 0.04$ & 0.312 & $0.13 \pm 0.07$ & $0.10 \pm 0.05$ & 0.197 \\
\hline Day 1 & $0.02 \pm 0.02$ & $0.01 \pm 0.01$ & 0.274 & $0.02 \pm 0.03$ & $0.01 \pm 0.02$ & 0.292 \\
\hline Day 4 & $0.01 \pm 0.03$ & $0.02 \pm 0.02$ & 0.110 & $0.05 \pm 0.08$ & $0.03 \pm 0.04$ & 0.002 \\
\hline Day 7 & $0.00 \pm 0.02$ & $0.02 \pm 0.04$ & 0.222 & $0.04 \pm 0.05$ & $0.05 \pm 0.02$ & 0.702 \\
\hline Day 28 & $0.00 \pm 0.01$ & $0.01 \pm 0.03$ & 0.694 & $0.06 \pm 0.03$ & $0.09 \pm 0.04$ & 0.114 \\
\hline \multicolumn{7}{|c|}{ Angular velocity steps, time constant (seconds) } \\
\hline Before & $3.69 \pm 1.67$ & $3.46 \pm 1.27$ & 0.690 & $4.01 \pm 1.26$ & $3.88 \pm 1.97$ & 0.826 \\
\hline Day 1 & $0.00 \pm 0.00$ & $0.00 \pm 0.00$ & 1 & $0.00 \pm 0.00$ & $0.00 \pm 0.00$ & 1 \\
\hline Day 4 & $0.22 \pm 0.54$ & $1.02 \pm 1.35$ & 0.176 & $1.19 \pm 0.63$ & $0.62 \pm 0.91$ & 0.336 \\
\hline Day 7 & $0.08 \pm 0.15$ & $1.11 \pm 1.62$ & 0.084 & $1.44 \pm 1.24$ & $1.77 \pm 1.53$ & 0.576 \\
\hline Day 28 & $1.21 \pm 0.58$ & $1.07 \pm 1.20$ & 0.807 & $2.52 \pm 1.68$ & $1.71 \pm 0.43$ & 0.170 \\
\hline
\end{tabular}

All values are represented as mean \pm SD (standard deviation). Statistically significant values are in bold. Static head tilt, Off-vertical-axis rotation (OVAR) and angular velocity steps values were each compared between UL and UVN mice populations, using a three-way repeated ANOVA and post-hoc Tukey test, all three model characteristics were $(100, p<0.0001)$

$U L$ unilateral labyrinthectomy, $U V N$ unilateral vestibular neurectomy

*Slope of a linear regression of both variables (vertical eye angle and head tilt degree) 
Table 4 Summary of differences between unilateral labyrinthectomy and unilateral vestibular neurectomy

\begin{tabular}{lll}
\hline Lesion & UL & UVN \\
\hline Surgical procedure length & Shortest $(16 \mathrm{~min})^{*}$ & Longest $(21 \mathrm{~min})^{*}$ \\
Mouse survival rate & Satisfactory $(86 \%)^{*}$ & Poor $(60 \%)^{*}$ \\
Cochlea & Intact & Destroyed \\
Vestibular labyrinth & Destroyed & Intact \\
Ganglion of Scarpa & Intact & Destroyed (suctioned) \\
Type of lesion & Preganglionic lesion & Postganglionic lesion \\
Degeneration of afferent fibres & No & Yes \\
Behavioural score & Shorter recovery & Longer recovery \\
Vestibular function & Similarly impaired & Similarly impaired \\
\hline
\end{tabular}

*No statistically significant difference paramount in both surgeries but especially UVN, to prevent dehydration (as mice are unable to drink on their own due to tumbling during the first $48 \mathrm{~h}$ ) but also to prevent ipsilateral keratitis if the facial nerve was damaged. The auditory function cannot be preserved due to the surgical destruction of the labyrinth, especially in the UVN procedure and its transcochlear approach (thus was not tested in this study).

UVN surgery seemed more severe and aggressive than UL: the behavioural score was significantly higher at day 4 and 7 and the number of nystagmus beats per minute was higher; however, vestibular compensation followed a comparable time course, both after 1 week and at 1 month.

Thus, for the same overall vestibular deficit, in our hands UL is preferred, as the procedure is shorter and bleeding and neurological per-operative risks are reduced with higher survival rates compared to UVN.

The destruction of the vestibular neurons in UVN (postganglionic lesion) induces Wallerian degeneration of the entire vestibular nerve and an inflammatory response reaching the vestibular nuclei $[21,40,41]$. Indeed degeneration of afferent fibres seems to take place after post- but not preganglionic lesions [42]. Such intense inflammation may be key in certain research protocols, in which case the choice of UVN over UL is justified. This has been shown in research on central vestibular neurogenesis, where the neurogenic potential has been identified after UVN but not after UL or pharmacological vestibular lesions [24, 43-45].

Although this was not the main objective of the study, the small number of mice limits the fine interpretation of functional results especially concerning ipsilesional and contralesional data. Some hypothesis may be drawn from our statistics, as contralesional stimulation partially recuperated in most cases at 1 month, in line with previous reports [14]. Concerning overall vestibular function after a unilateral vestibular lesion, this study confirms other papers showing a fast functional compensation period which corresponds to rapid behavioural improvement during the first post-operative week (especially during the first 3 days), which tends to stabilise with a prolonged hypofunction and near-normal behaviour at 1 month, except swimming which did not recuperate $[11,28]$. After initial poor bilateral MOR gain, gain improved in both UVN and UL mice at 1 month when the contralesional side was stimulated. The mechanism is unclear (it may reflect asymmetric otolith input to the central nervous system or interactions between semicircular canal-ocular and otolith-ocular reflexes) but it has been reported previously that the bias component remains either small or in an inappropriate direction during OVAR ipsilesional stimulation [46, 47]. Skew deviation is a well described vertical contralesional pupil elevation after peripheral vestibular lesions, whether of surgical, pharmacological or pathological origin $[48,49]$. Regarding vertical eye position during static head tilt, skew deviation limited the capacity to measure the response, especially during tilts towards the contralesional side from day 1 to 4 . Thus, the impairment was greatest at day 4 (UL) or 7 (UVN), and then partially recuperated. Lastly, concerning the canalar time constant measured during horizontal steps, there was no statistically significant difference between UL and UVN mice. However, UL contralesional values almost returned to perilesional values and were statistically different from ipsilesional values; whereas in UVN, ipsi- and contralesional values were not statistically different at 1 month. It is possible that the Wallerian degeneration of the nerve in UVN, inducing central vestibular nuclei inflammation, may impact velocity storage bilaterally, while UL remaining peripheral inputs allows for restoration of the response during rotation towards the contralesional side at 1 month. These hypotheses should be verified by dedicated studies.

\section{Conclusion}

UL and UVN are two reproducible surgical techniques to induce definite and total unilateral vestibular lesions. In both cases, surgical landmarks are key to quickly identify the vestibular system and protect blood vessels and the cranial cavity. These are mainly the facial nerve, the external auditory 
canal and the sternomastoid and digastric muscles. UVN and UL induce similar behavioural and functional vestibular deficits during a 1-month follow-up period. Thus, UL should be preferred in most cases as the procedure seems technically safer and quicker, with a higher survival rate. UVN can, however, be required in certain research protocols as the ganglion of Scarpa is destroyed, and Wallerian degeneration may spread inflammation to the central vestibular nuclei.

Acknowledgements This work was supported by the University of Paris and the CNRS. MB and FS received support from Marc Boulet Audition. CD received support from the Association Française d'Otologie et Oto-neurologie (AFON). This study contributes to the IdEx Université de Paris ANR-18-IDEX-0001.

This manuscript is part of a supplement supported by the German Federal Ministry of Education and Research within the funding initiative for integrated research and treatment centers.

\section{Compliance with ethical standards}

Conflicts of interest The authors do not have any commercial or other association that might pose a conflict of interest.

Open Access This article is licensed under a Creative Commons Attribution 4.0 International License, which permits use, sharing, adaptation, distribution and reproduction in any medium or format, as long as you give appropriate credit to the original author(s) and the source, provide a link to the Creative Commons licence, and indicate if changes were made. The images or other third party material in this article are included in the article's Creative Commons licence, unless indicated otherwise in a credit line to the material. If material is not included in the article's Creative Commons licence and your intended use is not permitted by statutory regulation or exceeds the permitted use, you will need to obtain permission directly from the copyright holder. To view a copy of this licence, visit http://creativecommons.org/licenses/by/4.0/.

\section{References}

1. Flourens P (1842) Recherches expérimentales sur les propriétés et les fonctions du système nerveux dans les animaux vertébrés. Ballière

2. Fluur E (1960) Vestibular compensation after labyrinthine destruction. Acta Otolaryngol 52:367-375. https://doi.org/10.3109/00016 486009123163

3. Precht W, Shimazu H, Markham CH (1966) A mechanism of central compensation of vestibular function following hemilabyrinthectomy. J Neurophysiol 29:996-1010. https://doi. org/10.1152/jn.1966.29.6.996

4. Smith PF, Curthoys IS (1989) Mechanisms of recovery following unilateral labyrinthectomy: a review. Brain Res Brain Res Rev 14:155-180. https://doi.org/10.1016/0165-0173(89)90013-1

5. Dieringer N (1995) "Vestibular compensation": neural plasticity and its relations to functional recovery after labyrinthine lesions in frogs and other vertebrates. Prog Neurobiol 46:97-129

6. Dieringer N (2003) Activity-related postlesional vestibular reorganization. Ann N Y Acad Sci 1004:50-60

7. Curthoys IS (2000) Vestibular compensation and substitution. Curr Opin Neurol 13:27-30. https://doi.org/10.1097/00019052200002000-00006

8. Cullen KE, Minor LB, Beraneck M, Sadeghi SG (2009) Neural substrates underlying vestibular compensation: contribution of peripheral versus central processing. J Vestib Res 19:171-182. https://doi.org/10.3233/VES-2009-0357

9. Beraneck M, Idoux E (2012) Reconsidering the role of neuronal intrinsic properties and neuromodulation in vestibular homeostasis. Front Neurol. https://doi.org/10.3389/fneur.2012.00025

10. Straka H, Zwergal A, Cullen KE (2016) Vestibular animal models: contributions to understanding physiology and disease. J Neurol 263(Suppl 1):S10-23. https://doi.org/10.1007/s00415-015-7909-y

11. Cassel R, Bordiga P, Carcaud J et al (2019) Morphological and functional correlates of vestibular synaptic deafferentation and repair in a mouse model of acute onset vertigo. Dis Model Mech. https://doi.org/10.1242/dmm.039115

12. Faralli A, Dagna F, Albera A et al (2016) Modifications of perineuronal nets and remodelling of excitatory and inhibitory afferents during vestibular compensation in the adult mouse. Brain Struct Funct 221:3193-3209. https://doi.org/10.1007/ s00429-015-1095-7

13. Yamaoka Y, Abe C, Morita H (2018) Comparison among ultrasonic, electrical apparatus, and toxic chemicals for vestibular lesion in mice. J Neurosci Methods 295:58-67. https://doi. org/10.1016/j.jneumeth.2017.11.021

14. Beraneck M, McKee JL, Aleisa M, Cullen KE (2008) Asymmetric recovery in cerebellar-deficient mice following unilateral labyrinthectomy. J Neurophysiol 100:945-958. https://doi. org/10.1152/jn.90319.2008

15. Hubner PP, Khan SI, Migliaccio AA (2017) The mammalian efferent vestibular system plays a crucial role in vestibulo-ocular reflex compensation after unilateral labyrinthectomy. J Neurophysiol 117:1553-1568. https://doi.org/10.1152/jn.01049.2015

16. Gacek RR, Khetarpal U (1998) Neurotrophin 3, not brainderived neurotrophic factor or neurotrophin 4, knockout mice have delay in vestibular compensation after unilateral labyrinthectomy. Laryngoscope 108:671-678. https://doi. org/10.1097/00005537-199805000-00009

17. Eron JN, Davidovics N, Della Santina CC (2015) Contribution of vestibular efferent system alpha- 9 nicotinic receptors to vestibulo-oculomotor interaction and short-term vestibular compensation after unilateral labyrinthectomy in mice. Neurosci Lett 602:156-161. https://doi.org/10.1016/j.neulet.2015.06.060

18. Aleisa M, Zeitouni AG, Cullen KE (2007) Vestibular compensation after unilateral labyrinthectomy: normal versus cerebellar dysfunctional mice. J Otolaryngol 36:315-321

19. Lambert FM, Straka H (2012) The frog vestibular system as a model for lesion-induced plasticity: basic neural principles and implications for posture control. Front Neurol 3:42. https://doi. org/10.3389/fneur.2012.00042

20. Pollack SM, Popratiloff A, Peusner KD (2004) Vestibular ganglionectomy and otolith nerve identification in the hatchling chicken. J Neurosci Methods 138:149-155. https://doi. org/10.1016/j.jneumeth.2004.04.023

21. Péricat D, Farina A, Agavnian-Couquiaud E et al (2017) Complete and irreversible unilateral vestibular loss: a novel rat model of vestibular pathology. J Neurosci Methods 283:83-91. https://doi.org/10.1016/J.JNEUMETH.2017.04.001

22. Li P, Ding D, Gao K, Salvi R (2015) Standardized surgical approaches to ear surgery in rats. J Otol 10:72-77. https://doi. org/10.1016/j.joto.2015.03.004

23. Hitier M, Sato G, Zhang Y et al (2016) Anatomy and surgical approach of rat's vestibular sensors and nerves. J Neurosci Methods 270:1-8. https://doi.org/10.1016/j.jneum eth.2016.05.013

24. Dutheil S, Brezun JM, Leonard J et al (2009) Neurogenesis and astrogenesis contribution to recovery of vestibular functions in the adult cat following unilateral vestibular neurectomy: cellular and behavioral evidence. Neuroscience 164:1444-1456. https:// doi.org/10.1016/J.NEUROSCIENCE.2009.09.048 
25. Gacek RR, Schoonmaker JE (1997) Morphologic changes in the vestibular nerves and nuclei after labyrinthectomy in the cat: a case for the neurotrophin hypothesis in vestibular compensation. Acta Otolaryngol 117:244-249. https://doi.org/10.3109/00016 489709117780

26. Schuknecht HF (1982) Behavior of the vestibular nerve following labyrinthectomy. Ann Otol Rhinol Laryngol Suppl 97:16-32

27. Faulstich M, van Alphen AM, Luo C et al (2006) Oculomotor plasticity during vestibular compensation does not depend on cerebellar LTD. J Neurophysiol 96:1187-1195. https://doi. org/10.1152/jn.00045.2006

28. Beraneck M, Cullen KE (2007) Activity of vestibular nuclei neurons during vestibular and optokinetic stimulation in the alert mouse. J Neurophysiol 98:1549-1565. https://doi.org/10.1152/ jn.00590.2007

29. Beraneck M, Bojados M, Le Seac'h A et al (2012) Ontogeny of mouse vestibulo-ocular reflex following genetic or environmental alteration of gravity sensing. PLoS ONE 7:e40414. https://doi. org/10.1371/journal.pone.0040414

30. França de Barros F, Carcaud J, Beraneck M (2019) Long-term sensory conflict in freely behaving mice. J Vis Exp. https://doi. org/10.3791/59135

31. Beraneck M, Lambert FM (2009) Impaired perception of gravity leads to altered head direction signals: what can we learn from vestibular-deficient mice? J Neurophysiol 102:12-14. https://doi. org/10.1152/jn.00351.2009

32. Stahl JS, Averbuch-Heller L, Leigh RJ (2000) Acquired nystagmus. Arch Ophthalmol 118:544-549. https://doi.org/10.1001/ archopht.118.4.544

33. Carcaud J, França de Barros F, Idoux E et al (2017) Long-lasting visuo-vestibular mismatch in freely-behaving mice reduces the vestibulo-ocular reflex and leads to neural changes in the direct vestibular pathway. eNeuro. https://doi.org/10.1523/ENEUR O.0290-16.2017

34. Idoux E, Tagliabue M, Beraneck M (2018) No gain no pain: relations between vestibulo-ocular reflexes and motion sickness in mice. Front Neurol 9:918. https://doi.org/10.3389/fneur .2018 .00918

35. Calabrese DR, Hullar TE (2006) Planar relationships of the semicircular canals in two strains of mice. J Assoc Res Otolaryngol 7:151-159. https://doi.org/10.1007/s10162-006-0031-1

36. Hess BJM, Dieringer N (1990) Spatial organization of the maculoocular reflex of the rat: responses during off-vertical axis rotation. Eur J Neurosci 2:909-919

37. Romand R, Krezel W, Beraneck M et al (2013) Retinoic acid deficiency impairs the vestibular function. J Neurosci 33:5856-5866. https://doi.org/10.1523/jneurosci.4618-12.2013
38. Oommen BS, Stahl JS (2008) Eye orientation during static tilts and its relationship to spontaneous head pitch in the laboratory mouse. Brain Res 1193:57-66. https://doi.org/10.1016/j.brain res.2007.11.053

39. Lenzarini F, Di Lascio N, Stea F et al (2016) Time course of isoflurane-induced vasodilation: a doppler ultrasound study of the left coronary artery in mice. Ultrasound Med Biol 42:999-1009. https://doi.org/10.1016/j.ultrasmedbio.2015.11.026

40. Gaudet AD, Popovich PG, Ramer MS (2011) Wallerian degeneration: gaining perspective on inflammatory events after peripheral nerve injury. J Neuroinflammation 8:110. https://doi. org/10.1186/1742-2094-8-110

41. Conforti L, Gilley J, Coleman MP (2014) Wallerian degeneration: an emerging axon death pathway linking injury and disease. Nat Rev Neurosci 15:394-409. https://doi.org/10.1038/nrn3680

42. Kunkel AW, Dieringer N (1994) Morphological and electrophysiological consequences of unilateral pre- versus postganglionic vestibular lesions in the frog. J Comp Physiol A 174:621-632. https://doi.org/10.1007/BF00217383

43. Dutheil S, Lacour M, Tighilet B (2011) Neurogenic potential of the vestibular nuclei and behavioural recovery time course in the adult cat are governed by the nature of the vestibular damage. PLoS ONE 6:e22262. https://doi.org/10.1371/journal.pone.00222 62

44. Dutheil S, Escoffier G, Gharbi A et al (2013) GABA(A) receptor agonist and antagonist alter vestibular compensation and different steps of reactive neurogenesis in deafferented vestibular nuclei of adult cats. J Neurosci 33:15555-15566. https://doi.org/10.3389/ fnint.2013.00087

45. Tighilet B, Chabbert C (2019) Adult neurogenesis promotes balance recovery after vestibular loss. Prog Neurobiol 174:28-35. https://doi.org/10.1016/j.pneurobio.2019.01.001

46. Furman JM, Schor RH, Kamerer DB (1993) Off-vertical axis rotational responses in patients with unilateral peripheral vestibular lesions. Ann Otol Rhinol Laryngol 102:137-143. https://doi. org/10.1177/000348949310200211

47. Sugita-Kitajima A, Koizuka I (2014) Evaluation of the vestibuloocular reflex using sinusoidal off-vertical axis rotation in patients with canal paresis. Auris Nasus Larynx 41:22-26. https://doi. org/10.1016/j.anl.2013.04.014

48. Vibert D, Häusler R, Safran AB, Koerner F (1996) Diplopia from skew deviation in unilateral peripheral vestibular lesions. Acta Otolaryngol 116:170-176. https://doi.org/10.3109/0001648960 9137816

49. Ng D, Fouladvand M, Lalwani AK (2011) Skew deviation after intratympanic gentamicin therapy. Laryngoscope 121:492-494. https://doi.org/10.1002/lary.21279 